\title{
Type 2 diabetes in children: recognition and complications
}

\author{
“...understanding of the true prevalence of Type 2 diabetes \\ mellitus in adolescents is complicated by uncertainties in \\ diagnosis and variations in the definition."
}

\section{Prevalence}

The epidemiology of Type 2 diabetes mellitus (T2DM) in adolescents in the USA and worldwide has been reviewed multiple times [1-3]. These reviews confirm the rise in the global incidence of T2DM in children and adolescents over the last two decades and suggest a close relationship between an increase in obesity in the general population, an increase in the rate of T2DM in adults and the later appearance of the disorder in adolescents [2]. There have been few recent population-based studies of Type 2 diabetes in adolescents since these reviews were published. The SEARCH for Diabetes in Youth Study is a six-center population-based ascertainment of physician-diagnosed diabetes in adolescents of less than 20 years of age in the USA. Among older adolescents, the proportion of T2DM ranged from $6 \%$ of new cases of diabetes $(0.19$ cases per 1000 adolescents for non-Hispanic white adolescents) to $76 \%$ (1.74 cases per 1000 adolescents for American-Indian adolescents) [4].

However, understanding the true prevalence of T2DM in adolescents is complicated by uncertainties in diagnosis and variations in the definition. T2DM is most rigorously defined as diabetes accompanied by obesity, evidence of insulin resistance, relative insulin deficiency and the absence of pancreatic autoimmunity. However, reported series have frequently relied on the clinical diagnosis of Type 2 diabetes in the obese minority of children who have short- to long-term independence from insulin requirements. In such cohorts, as many as $15 \%$ of the adolescents are found to exhibit evidence of autoimmunity [5-9], creating a category of T2DM patients with autoimmunity that challenges our understanding of the true prevalence of the disorder. This has prompted some authors to propose the concept of 'Type 1.5', 'double' or 'hybrid' diabetes.

The concept that such patients represent a 'new' type of diabetes has been prevalent in the lay media, but obscures the underlying pathophysiology of the disorder. Instead, this group of adolescents is best understood as having autoimmune Type 1 diabetes mellitus (T1DM), which presents on the background of an obese, insulin-resistant phenotype. Unfortunately, being overweight and of minority ethnicity does not protect children from the development of T1DM. Therefore, as the pediatric population becomes more obese and more likely to be of an ethnic minority, more children presenting with T1DM will be, like their peers, more obese and more likely to be African-American or Hispanic. Yet these antibody-positive individuals are significantly less overweight and younger than antibodynegative patients [10], and they have higher hemoglobin A1c (HbA1c) concentrations and a greater degree of $\beta$-cell impairment [11,12], all of which result in the more rapid development of insulin dependence [10].

Therefore, the diabetes course of these antibody-positive individuals is ultimately more similar to that of individuals with T1DM and the rapid progression to insulin dependence in these patients must be taken into account in their treatment - from the perspective of their diabetes care, these individuals are best understood to have T1DM. At the same time, the presence of obesity and insulin resistance confers the expected risks of hypertension, dyslipidemia, sleep disturbance and hepatic steatosis. While both the etiology of their diabetes and the consequences conferred by obesity contribute to our treatment decisions and long-term risk, conceptualizing a new entity to describe these individuals serves no purpose except to undermine our understanding of the pathophysiology and implications of $\beta$-cell impairment and insulin resistance.

\section{Screening}

Despite the increasing prevalence of clinical T2DM among adolescents, the prevalence of undiagnosed diabetes among adolescents remains unresolved. Studies have reported
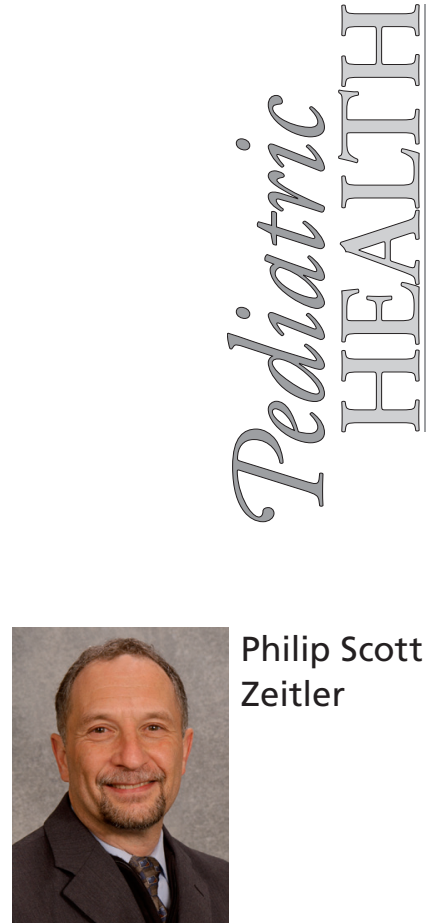

Philip Scott Zeitler

Division of Endocrinology, Department of Pediatrics, University of Colorado Denver, CO 80045, USA

Tel.: +1 7207776128

Fax: +1 7207777301

phil.zeitler@ucdenver.edu

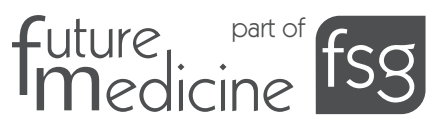


undiagnosed T2DM in $1-4 \%$ of adolescents presenting to an obesity clinic for evaluation, while population-based screenings of high-risk adolescents in Europe have reported rates from 0 to $6 \%$, depending on the criteria used for selection of the population (reviewed in [13]).

"Given the low prevalence of undiagnosed diabetes in the adolescent population ... there is little evidence to support the widespread population-based screening of asymptomatic adolescents based on broad indicators of increased risk."

In the general population, undiagnosed cases of T2DM appear to be much rarer than what was originally anticipated. In a nationally representative sample of 915 US adolescents 12-19 years of age who were examined in the 1999-2000 National Health and Nutrition Examination Survey (NHANES), no cases of T2DM were identified, although the prevalence of impaired fasting glucose (IFG; fasting glucose levels between 100 and $125 \mathrm{ng} / \mathrm{dl}$ ) was $7.0 \%$ and higher in boys than in girls, and reached nearly $18 \%$ in obese adolescents [14]. Similarly, in a study at 12 middle schools around the USA involving 1740 eighth graders of whom $49 \%$ had a BMI greater than the 85 th percentile, IFG was present in $40.5 \%$, but diabetes by the fasting criteria was identified in only $0.4 \%$. Following a glucose load, only $0.1 \%$ had diabetes [15]. Notably, the presence of IFG was not a strong predictor of diabetes by the oral glucose tolerance test (OGTT) in this sample.

Given the low prevalence of undiagnosed diabetes in the adolescent population, even among those identified as high-risk individuals owing to factors such as ethnicity or family history, there is little evidence to support the widespread population-based screening of asymptomatic adolescents based on broad indicators of increased risk. However, guidance from the literature for identifying individuals for whom further testing is most likely to yield clinically relevant results is limited.

To complicate the issue further, Libman and colleagues have reported that the agreement between a first and second OGTT performed within 1 month of each other is only 22 and $27 \%$ for IFG and impaired glucose tolerance (IGT; 2-h glucose level between 140 and $199 \mathrm{mg} / \mathrm{dl}$ ), respectively [16]. Interestingly, those adolescents with discordant OGTT results were more likely to demonstrate evidence of subtle abnormalities in glucose metabolism, suggesting that even if they do not reproducibly meet the specific criteria, adolescents who have at least one abnormal OGTT are likely to experience early dysfunction of glucose metabolism.

Recent guidelines issued by the International Society for Pediatric and Adolescent Diabetes (ISPAD) recommend testing for IFG, IGT and diabetes in children with a BMI between the 85th and 95th percentiles who have a family history of cardiovascular disease and/or signs of insulin resistance, and in all children with a BMI greater than the 95th percentile [9]. However, the evidence noted above suggests that the value of an OGTT for the diagnosis of glucose dysregulation in an asymptomatic adolescent is too limited to support these recommendations. Given the low prevalence of undiagnosed T2DM, even among high-risk individuals, a large number of OGTTs would have to be performed to identify a single case that is not clinically apparent. Furthermore, an OGTT in this population is much more likely to identify IFG or IGT than T2DM, yet we currently have no information regarding the benefits of identifying these prediabetic states in the adolescent population. Finally, the poor reproducibility of the OGTT implies that the identification of abnormal glucose regulation using a single test is unreliable and thus, repeated testing would be required. Therefore, adherence to these guidelines would lead to the performance of a large number of OGTTs that would identify a very small number of cases of T2DM but would, in the process, result in the identification of many cases of prediabetes that we do not know how to treat. A much deeper appreciation of the likely outcome of extensive screening for diabetes and a clearer understanding of risk among adolescents is necessary before truly useful guidelines can be developed.

\section{Treatment}

While there has been increasing attention to the emergence of T2DM in the adolescent population, studies remain limited regarding the best approach to treatment. For the most part, pediatric diabetologists have had to rely on treatment paradigms derived from research and their experience of the care of adults with T2DM $[17,18]$. There are only two published randomized controlled trials of 
oral hypoglycemic agents in adolescents. In a 16-week randomized placebo-controlled multicenter trial of 82 adolescents, Jones and colleagues reported that metformin was safe and effective, with improvements having been observed in both fasting plasma glucose and mean $\mathrm{HbAlc}$ values compared with the placebo group [19]. However, the drop-out rate in this study was $55 \%$ in the metformin arm and $93 \%$ in the placebo arm. Based on these data, metformin, which was approved for use in pediatric T2DM. In another trial, 285 adolescents completed a 24-week comparison of glimeperide and metformin that demonstrated that glimepiride decreased $\mathrm{HbAlc}$ levels to a degree that is equivalent to that of metfor$\min$ [20]. However, glimeperide was associated with more weight gain and more frequent episodes of mild and moderate hypoglycemia than metformin. There have been no published trials of the use of other agents. Indeed, there have been no long-term trials reported examining the safety and efficacy of insulin in adolescent patients with T2DM [18]. To date, metformin remains the only agent to be specifically approved for the treatment of Type 2 diabetes in adolescents.

The Treatment Options for Type 2 Diabetes in Youth (TODAY) study is a 15-center $\mathrm{NIH}$-supported randomized clinical trial, comparing the long-term safety and efficacy of metformin alone and in combination with rosiglitazone, and intensive lifestyle modification in adolescents with T2DM, and has recently finished enrollment of 700 adolescents with rigorously-defined T2DM [21]. When the data are complete in late 2011, the TODAY study will provide critical information regarding the determinants of long-term glucose control in adolescents, as well as the first large-scale insight into the natural history of T2DM in adolescents, including changes over time in body composition, comorbidities, insulin secretion and sensitivity, activity and fitness, diet and the economic costs of diabetes for these adolescents and their families.

\section{Complications}

Like adults, adolescents with T2DM have a higher prevalence of comorbidities, such as hypertension, hyperlipidemia, nonalcoholic fatty liver disease and metabolic syndrome, which are associated with increased cardiovascular morbidity and mortality. Furthermore, these comorbidities are substantially more prevalent at diagnosis in adolescents with
T2DM than in those with T1DM and the progression of end-organ damage is much more rapid, including the development of microand macroalbuminuria, and proliferative retinopathy [22].

Among patients with T2DM in the SEARCH for Diabetes in Youth Study, 33\% had a total cholesterol (TC) concentration of greater than $200 \mathrm{mg} / \mathrm{dl}, 24 \%$ had a low-density lipoprotein cholesterol (LDL-C) concentration greater than $130 \mathrm{mg} / \mathrm{dl}, 29 \%$ had a triglycerides concentration greater than $150 \mathrm{mg} / \mathrm{dl}$ and $44 \%$ had a high-density lipoprotein cholesterol (HDL-C) concentration of less than $40 \mathrm{mg} / \mathrm{dl}$. The percentages for T1DM were 19, 15 and $12 \%$, respectively. Most importantly, in this cohort, only $1 \%$ of adolescents were receiving pharmacologic therapy for dyslipidemia [23]. In another study from the same group involving adolescents with Type 2 diabetes, $36 \%$ had elevated apolipoprotein B levels and 36\% had dense LDL, but only $23 \%$ had elevated LDL-C concentrations, and among adolescents with poorly controlled T2DM, 72\% had elevated apolipoprotein B levels and $62 \%$ had dense LDL [24].

Among adolescents with T2DM, 92\% had two or more cardiovascular risk factors, including abnormal lipids, a large waist circumference and hypertension [25]. Furthermore, when compared with matched control adolescents, those with T2DM had a higher prevalence of elevated blood pressure, obesity, a large waist circumference, low HDL-C levels, high triglyceride levels and a high albumin:creatinine ratio, as well as inflammatory markers such as fibrinogen, IL- 6 and C-reactive protein, along with lower adiponectin levels [26].

“Like adults, adolescents with T2DM have a higher prevalence of comorbidities, such as hypertension, hyperlipidemia, nonalcoholic fatty liver disease and metabolic syndrome, which are associated with increased cardiovascular morbidity and mortality."

Information regarding overt cardiovascular complications in adolescents with T2DM has been limited and has included reports of increased night-time systolic and diastolic pressure and diminished nocturnal decline in blood pressure, increased posterior and septal wall thickness, left ventricular hypertrophy and 
increased aortic pulse wave velocity (reviewed in [22]). More recent reports have confirmed and extended these findings. Girls with T2DM had larger left ventricle (LV) dimensions and LV masses along with impairment of diastolic filling and systolic longitudinal function compared with lean and overweight nondiabetic subjects. Half of the group met the published criteria for LV hypertrophy and LV dilatation and 25\% demonstrated evidence of elevated LV filling pressure in association with structural abnormalities [27]. In addition, both the maximal heart rate and exercise stroke volume were significantly reduced in girls with T2DM [28]. The possibility of progressive atherosclerosis and cardiac impairment leading to clinically significant cardiovascular morbidity and mortality in young adulthood is sobering.

Consensus guidelines for the treatment of comorbidities in high-risk pediatric patients have been developed based on the effectiveness of intervention in adults and limited pediatric data [29], but these guidelines are based solely on expert opinion. There is a serious lack of long-term data regarding the potential benefits of the early initiation of adjunctive therapies in adolescents with T2DM with regard to morbidity and mortality. Furthermore, pediatricians may be more hesitant to treat adolescents with chronic medications owing to their young age and long-term exposure to side effects. Therefore, in the absence of convincing evidence of efficacy, treatment of comorbidities is often delayed or omitted [23].

\section{“Type 2 diabetes and other disorders associated with rising rates of adolescent obesity will rank among the most costly and potentially devastating public health risks of the next 50 years."}

However, robust evidence supporting the treatment of these comorbidites may never become available. Studies examining the efficacy of treatment for comorbidities, such as hypertension and dyslipidemia, in adolescents with T2DM will need to be of long duration and large size. More importantly, a randomized trial of intervention compared with nonintervention may be unethical, given the evidence for the efficacy of comorbidity treatment in adults. Therefore, it is highly probable that pediatric diabetologists will need to continue to extrapolate evidence from adult studies to identify an appropriate balance between the timing of initiation of potentially beneficial interventions and the risk of side effects.

\section{Future perspective}

Type 2 diabetes and other disorders associated with rising rates of adolescent obesity will rank among the most costly and potentially devastating public health risks of the next 50 years. Given the prevalence of the disorder in developed countries, the expected wave of diabetes among adolescents in the rapidly developing world, the increased risk for obesity and diabetes among the off-spring of women who develop diabetes in adolescence and the high rates of diabetesrelated complications that are being reported, it is likely that the next few generations of young individuals will experience high rates of morbidity and a shortened life expectancy. For those adolescents who are already affected by Type 2 diabetes, there is no time to wait for the results of controlled trials to determine the optimal approaches to the treatment of diabetes and comorbidities; treatment will need to be guided by experience in adults. Meanwhile, healthcare systems must be prepared to initiate timely secondary prevention strategies in order to limit complications in affected young adults, many of whom will have limited or no reliable sources of healthcare, as well as to care for the inevitable increase in serious morbidities such as kidney failure, amputation and heart disease. At the same time, it will be critical that investments are made in improving our understanding of the complex biological, social, psychological and economic factors that contribute to this phenomenon. Finally, health authorities and the public will need to develop the will and commitment to recognize this problem, which disproportionately affects minority and economically disadvantaged populations most directly, as a major public health challenge that requires a comprehensive, and seemingly costly, response if we are to avoid a future in which large portions of the young adult population are burdened with disease.

\section{Financial \& competing interests disclosure}

The author has no relevant affliations or financial involvement with any organization or entity with a financial interest in or financial conflict with the subject matter or materials discussed in the manuscript. This includes employment, consultancies, honoraria, stock ownership or options, expert testimony, grants or patents received or pending, or royalties.

No writing assistance was utilized in the production of this manuscript. 


\section{Bibliography}

1. Fagot-Campagna A, Pettitt DJ, Engelgau MM et al.: Type 2 diabetes among north american children and adolescents: an epidemiological review and public health perspective. J. Pediatr. 136, 664-672 (2000).

2. Pinhas-Hamiel O, Zeitler P: The global spread of Type 2 diabetes in children and adolescents. J. Pediatr. 145, 693-700 (2005).

3. Nadeau K, Dabelea D: Epidemiology of Type 2 diabetes in children and adolescents. In: Epidemiology of Pediatric and Adolescent Diabetes. Dabelea D, Klingensmith GJ (Eds). Informa Healthcare, NY, USA 103-124 (2008).

4. SEARCH for Diabetes in Youth Study Group; Liese AD, D'Agostino R Jr, Hamman RF et al.: The burden of diabetes mellitus among US youth: prevalence estimates from the SEARCH for Diabetes in Youth Study. Pediatrics 118, 1510-1518 (2006).

5. Hathout EH, Thomas W, El-Shahawy M, Nahab F, Mace JW: Diabetic autoimmune markers in children and adolescents with Type 2 diabetes. Pediatrics 107, E102 (2001).

6. Umpaichitra V, Banerji MA, Castells S: Autoantibodies in children with Type 2 diabetes mellitus. J. Pediatr. Endocrinol. Metab. 15, 525-230 (2002).

7. Reinehr T, Schober E, Wiegand S, Thon A, Holl RW; DPV-Wiss Study Group: $\beta$-cell autoantibodies in children with Type 2 diabetes mellitus: subgroup or misclassification? Arch. Dis. Child. 91, 473-477 (2006).

8. Levitt Katz LE, Jawad AF, Ganesh J, Abraham M, Murphy K, Lipman TH: Fasting C-peptide and insulin like growth factor binding protein-one levels help to distinguish childhood Type 1 and Type 2 diabetes in diagnosis. Pediatr. Diabetes 8, 53-59 (2007).

9. Rosenbloom AL, Silverstein JH, Amemiya $S$, Zeitler P, Klingensmith GJ: ISPAD Clinical Practice Consensus Guideline (2006)-2007. Type 2 diabetes mellitus in the child and adolescent. Pediatr. Diabetes 9, 512-526 (2008).

10. Turner R, Stratton I, Horton V et al: : Autoantibodies to islet cell cytoplasm and glutamic acid decarboxylase for prediction of insulin requirement in Type 2 diabetes UKPDS 25. Lancet 350, 1288-1293 (1997).

11. Tfayli H, Bacha F, Gungor N, Arslanian S: Phenotypic Type 2 diabetes in obese youth: insulin sensitivity and secretion in islet-cell antibody negative vs. antibody positive patients. Diabetes 58(3), 738-744 (2009).
12. Klingensmith GJ, Coombs LP, Arslanian S et al.: Antibody positivity in subjects screened for participation in a treatment trial for T2D in youth. Presented at: The 66th Scientific Session of the American Diabetes Association. Washington DC, USA, 9-13 June 2006.

13. Zeitler P, Pinhas-Hamiel O: Prevention and screening for Type 2 diabetes in youth. In: Epidemiology of Pediatric and Adolescent Diabetes. Dabelea D, Klingensmith GJ (Eds). Informa Healthcare, NY, USA 201-216 (2008).

14. Williams DE, Cadwell BL, Cheng YJ et al.: Prevalence of impaired fasting glucose and its relationship with cardiovascular disease risk factors in US adolescents, 1999-2000. Pediatrics 116, 1122-1126 (2005).

15. Baranowski T, Cooper DM, Harrell J et al.: Presence of diabetes risk factors in a large U.S. eighth-grade cohort. Diabetes Care 29, 212-217 (2006).

16. Libman IM, Barinas-Mitchell E, Bartucci A, Robertson R, Arslanian S: Reproducibility of the oral glucose tolerance test in overweight children. J. Clin. Endocrinol. Metab. 93, 4231-4237 (2008).

17. Pinhas-Hamiel O, Zeitler P: Advances in the epidemiology and treatment of Type 2 diabetes in children. Adv. Pediatr. 52, 223-259 (2005).

18. Pinhas-Hamiel O, Zeitler P: Clinical presentation and treatment of Type 2 diabetes in children. Pediatr. Diabetes 8(Suppl. 9), 16-27 (2007).

19. Jones KL, Arslanian S, Peterokova VA, Park JS, Tomlinson MJ: Effect of metformin in pediatric patients with Type 2 diabetes: a randomized controlled trial. Diabetes Care 25, 89-94 (2002).

20. Gottschalk M, Danne T, Vlajnic A, Cara JF: Glimepiride versus metformin as monotherapy in pediatric patients with Type 2 diabetes: a randomized, single-blind comparative study. Diabetes Care 30, 790-794 (2007).

21. The TODAY Study Group; Zeitler P, Epstein L, Hirst K et al.: Treatment options for Type 2 diabetes in adolescents and youth: a study of the comparative efficacy of metformin alone or in combination with rosiglitazone or lifestyle intervention in adolescents with Type 2 diabetes. Pediatr. Diabetes 8, 74-87 (2007).

22. Pinhas-Hamiel O, Zeitler P: Acute and chronic complications of Type 2 diabetes mellitus in children and adoelscents. Lancet 369, 1823-1831 (2007).
23. Kershnar AK, Daniels SR, Imperatore G et al.: Lipid abnormalities are prevalent in youth with Type 1 and Type 2 diabetes: the SEARCH for Diabetes in Youth Study. J. Pediatr. 149, 314-319 (2006).

24. Albers JJ, Marcovina SM, Imperatore G et al.: Prevalence and determinants of elevated apolipoprotein B and dense low-density lipoprotein in youths with Type 1 and Type 2 diabetes. J. Clin. Endocrinol. Metab. 93, 735-742 (2008).

25. Rodriguez BL, Fujimoto WY, Mayer-Davis EJ et al:: Prevalence of cardiovascular disease risk factors in U.S. children and adolescents with diabetes: the SEARCH for diabetes in youth study. Diabetes Care 29, 1891-1896 (2006).

26. West NA, Hamman RF, Mayer-Davis EJ et al: Cardiovascular risk factors among youth with and without Type 2 diabetes: differences and possible mechanisms. Diabetes Care 32, 175-180 (2009).

27. Whalley GA, Gusso S, Hofman P et al: Structural and functional cardiac abnormalities in adolescent girls with poorly-controlled Type 2 diabetes mellitus. Diabetes Care 32(5), 883-888 (2009).

28. Gusso S, Hofman P, Lalande S, Cutfield W, Robinson E, Baldi JC: Impaired stroke volume and aerobic capacity in female adolescents with Type 1 and Type 2 diabetes mellitus. Diabetologia 51, 1317-1320 (2009).

29. Kavey RE, Allada V, Daniels SR et al.: Cardiovascular risk reduction in high-risk pediatric patients: a scientific statement from the American Heart Association Expert Panel on Population and Prevention Science; the Councils on Cardiovascular Disease in the Young, Epidemiology and Prevention, Nutrition, Physical Activity and Metabolism, High Blood Pressure Research, Cardiovascular Nursing, and the Kidney in Heart Disease; and the Interdisciplinary Working Group on Quality of Care and Outcomes Research: endorsed by the American Academy of Pediatrics. Circulation 114, 2710-2738 (2006).

\section{Affiliation}

- $\quad$ Philip Scott Zeitler Division of Endocrinology, Department of Pediatrics, University of Colorado Denver, CO 80045, USA

Tel.: +1 7207776128

Fax: +1 7207777301

phil.zeitler@ucdenver.edu 